\title{
Hidradenitis Suppurativa Management in the United States: An Analysis of the National Ambulatory Medical Care Survey and MarketScan Medicaid Databases
}

\author{
Scott A. Davis ${ }^{a}$ Hsien-Chang Lin ${ }^{d}$ Rajesh Balkrishnan ${ }^{\text {e }}$ Steven R. Feldman ${ }^{a-c}$ \\ ${ }^{a}$ Center for Dermatology Research, Department of Dermatology, and Departments of ${ }^{b}$ Pathology and ${ }^{c}$ Public \\ Health Sciences, Wake Forest School of Medicine, Winston-Salem, N.C., d Department of Applied Health \\ Science, School of Public Health, Indiana University, Bloomington, Ind., and e Department of Clinical, Social and \\ Administrative Sciences, College of Pharmacy, University of Michigan, Ann Arbor, Mich., USA
}

\section{Key Words}

Apocrine glands · Antibiotics · Biologics · Tumor necrosis factor inhibitors · National Ambulatory Medical Care Survey . Demographics

\begin{abstract}
Purpose: To present nationally representative data demonstrating how frequently hidradenitis suppurativa (HS) occurs in specific groups and how it is currently managed. Methods: We analyzed data from the 1990-2009 National Ambulatory Medical Care Survey (NAMCS) and the 2003-2007 MarketScan Medicaid databases for patients with a diagnosis of HS (ICD-9-CM code 705.83). Visits per 100,000 population of each race and ethnicity were calculated using the 2000 US Census data for specific demographics. Results: There were 164,000 patient visits (95\% Cl: 128,000-200,000) annually with a diagnosis of HS in the NAMCS, and 17,270 HS patients were found in the MarketScan Medicaid over the 5-year period. Antibiotics were the most common treatment, followed by pain medications, topical steroids, and isotretinoin. Prescriptions of biologics and systemic methotrexate, cyclosporine, and acitretin were not observed in the NAMCS. Physicians prescribed medications in $74 \%$ of visits and used
\end{abstract}

procedures in $11 \%$ of visits. African Americans, females, and young adults had higher numbers of visits for HS. Conclusions: Our data showing a maximum of $0.06 \%$ of the population being treated for HS in a given year are consistent with the low estimates of HS prevalence. Compared to the current prescribing patterns, the more frequent prescription of biologics and systemic treatments may yield better outcomes.

(c) 2015 S. Karger AG, Basel

\section{Introduction}

Hidradenitis suppurativa (HS) is a condition characterized by lesions affecting the skin with apocrine glands [1]. Treatments may include topical or oral antibiotics, topical or systemic steroids, isotretinoin, antiandrogens in some female patients, cyclosporine, and tumor necrosis factor inhibitors as well as surgery and laser therapy [1]. Few randomized controlled trials have been performed to evaluate the efficacy of HS therapies, although etanercept, adalimumab, and infliximab have all been tested in randomized controlled trials, with mixed results [2-4]. Although there are no official guidelines for treating HS, antibiotics tend to be preferred for mild to mod-

\section{KARGER 125}

(c) 2015 S. Karger AG, Base

$2296-9195 / 15 / 0012-0065 \$ 39.50 / 0$

E-Mail karger@karger.com

www.karger.com/sad
Steven R. Feldman, $\mathrm{MD}, \mathrm{PhD}$

Department of Dermatology, Wake Forest School of Medicine

Medical Center Boulevard

Winston-Salem, NC 27157-1071 (USA)

E-Mail sfeldman@wakehealth.edu 
erate cases, while more severe cases may be treated with any of several systemic therapies such as steroids and biologics [1]. This study attempts to present nationally representative data to demonstrate how frequently HS occurs in specific groups and how it is currently managed.

\section{Methods}

The National Ambulatory Medical Care Survey (NAMCS), conducted by the National Center for Health Statistics (NCHS), is designed to meet the need for objective, reliable information about the provision and use of ambulatory medical care services in the United States. Findings are based on a stratified random sample of visits to nonfederally employed office-based physicians who are primarily engaged in direct patient care. Physicians in the specialties of anesthesiology, pathology, and radiology, which are not normally involved in direct patient care, are excluded from the survey. The survey was conducted annually from 1973 to 1981, in 1985, and annually since 1989.

Each physician is randomly assigned to a 1-week reporting period in which data for a systematic random sample of visits are recorded by the physician, office staff, or an NCHS survey staff member on an encounter form provided for that purpose. Data are obtained on patients' symptoms, physicians' diagnoses, medications ordered or provided, and the demographic characteristics of patients and services provided, including information on diagnostic procedures, patient management, and planned future treatment. Survey weights are applied to estimate visit counts. Estimated quantities of visits were rounded to the same number of significant digits as in the unweighted counts.

For the current study, we searched the 1990-2009 NAMCS for visits with a primary, secondary, or tertiary diagnosis of HS (ICD-9 code 705.83). Frequency tables were produced for demographic data, reasons for visit, medications, medication classes, procedures, and comorbidities associated with HS. Drug mentions include medications administered, prescribed, dispensed, or discussed at the visit. Linear regressions (SAS PROC SURVEYREG) were used to analyze trends over time in demographics and the treatment of HS. All data analyses were performed with SAS 9.1.3 (SAS Institute, Cary, N.C., USA) and Stata 12 (Stata, College Station, Tex., USA). To calculate estimates for the number of visits per 100,000 population of each race and ethnicity, we used data from the 2000 US Census on the population for each race and ethnicity.

The MarketScan Medicaid database is a family of databases containing individual-level health care claims, lab test results, and hospital discharge information from large employers, managed care organizations, hospitals, and public health insurance programs, such as Medicare and Medicaid. For this study, the dataset for Medicaid enrollees was used. Overall, the database covers 100 million patients in the United States and captures the full continuum of care in all health care settings, including physician office visits, hospital admissions, carve-out care, as well as retail, mail order, and specialty pharmacies. Patients with at least one outpatient visit during the time period of 2003-2007 were included in this study. We identified the date of the first diagnosis of HS for each patient, and only the medication records after the first diagnosis of HS were included in the analysis.
Table 1. Leading reasons for visit in NAMCS patient with a diagnosis of HS

\section{Visits, \%}

Reason for visit (primary, secondary, or tertiary)

Carbuncle, furuncle, boil, cellulitis, or abscess, NEC 14.2

Other diseases of the skin $\quad 13.0$

Postoperative visit $\quad 12.5$

Other growths of the skin $\quad 9.9$

Lump/mass/tumor of arm 6.3

Skin lesion, NOS $\quad 6.3$

Skin rash $\quad 5.6$

Headache $\quad 4.3$

Pain/ache/soreness/discomfort of arm $\quad 4.1$

Swollen or enlarged lymph glands 3.9

Reason for visit (primary reason only)

Carbuncle, furuncle, boil, cellulitis, abscess, NEC $\quad 13.5$

Other growths of the skin $\quad 8.8$

Other diseases of the skin $\quad 6.8$

Lump/mass/tumor of arm $\quad 5.6$

Postoperative visit $\quad 5.3$

Skin lesion, NOS $\quad 4.8$

Pain/ache/soreness/discomfort of arm $\quad 3.7$

Swollen or enlarged lymph glands $\quad 3.5$

Acne or pimples $\quad 3.5$

Infection of skin, NOS $\quad 2.8$

Patients most often complained of a carbuncle, furuncle, boil, cellulitis, or abscess. Totals may add to $>100 \%$ as patients may have up to three reasons for visit. NEC $=$ Not elsewhere classified; NOS $=$ not otherwise specified.

\section{Results}

In the NAMCS, there were an estimated 3,280,000 visits (95\% CI: 2,560,000-4,000,000; fig. 1; table 1) over the period 1990-2009 with a diagnosis of HS (ICD-9 code 705.83). Of these, HS was the primary diagnosis for $2,610,000$, the secondary diagnosis for 500,000 , and the tertiary diagnosis for 160,000 . It was the sole diagnosis for $1,820,000$ or $55.5 \%$ of the HS visits. On an annual basis, there were an average of $164,000 \mathrm{HS}$ visits per year (95\% CI: 128,000-200,000). In the MarketScan Medicaid dataset, there were 17,270 patients with a diagnosis of HS over the 5-year period from 2003 to 2007. Of these, 15,959 had a record of claims after their first HS diagnosis.

The leading reasons for visit among the patients with a diagnosis of HS in the NAMCS included carbuncle/furuncle/boil/cellulitis/abscess not elsewhere classified, other diseases of the skin (including HS, as there is no
Davis/Lin/Balkrishnan/Feldman 
Table 2. Dermatologists and nondermatologists in the NAMCS differ in treatment patterns for HS

\begin{tabular}{lccc}
\hline & All visits & Dermatologists & Nondermatologists \\
\hline Medication only & $2,180,000(66)$ & $580,000(78)$ & $1,600,000(63)$ \\
Procedure only & $120,000(4)$ & $40,000(5)$ & $80,000(3)$ \\
Medication + procedure & $260,000(8)$ & $110,000(15)$ & $150,000(6)$ \\
No treatment & $730,000(22)$ & $10,000(2)$ & $710,000(28)$ \\
Total number of medications & $2,430,000(74)$ & $690,000(94)$ & $1,740,000(69)$ \\
Total number of procedures & $380,000(11)$ & $150,000(20)$ & $230,000(9)$ \\
\hline
\end{tabular}

Dermatologists used a procedure in $20 \%$ of visits and a medication in almost all visits, while nondermatologists appeared to use both types less often. Figures in parentheses indicate frequency of visit in percentages.

Table 3. Leading treatments for HS in the NAMCS included several antibiotics as well as steroids such as triamcinolone

\begin{tabular}{lcc}
\hline Medication & Frequency & Visits, \% \\
\hline Leading medications for diagnosis & $(1$ st, 2nd, or 3 rd) & of HS in \\
NAMCS & & \\
Minocycline & 300,000 & 9.2 \\
Cephalexin & 250,000 & 7.6 \\
Ciprofloxacin & 230,000 & 6.9 \\
Triamcinolone & 220,000 & 6.7 \\
Clindamycin & 150,000 & 4.5 \\
Erythromycin & 150,000 & 4.4 \\
Benzoyl peroxide & 140,000 & 4.3 \\
Albuterol & 130,000 & 4.0 \\
Isotretinoin & 120,000 & 3.8 \\
Acetaminophen/oxycodone & 110,000 & 3.5 \\
\hline Leading medications for sole diagnosis of HS in NAMCS \\
Triamcinolone & 150,000 & 8.4 \\
Acetaminophen/oxycodone & 110,000 & 6.2 \\
Ciprofloxacin & 100,000 & 5.7 \\
Cephradine & 90,000 & 5.1 \\
Isotretinoin & 90,000 & 4.7 \\
Minocycline & 80,000 & 4.5 \\
Doxycycline & 70,000 & 3.9 \\
Clindamycin & 70,000 & 3.8 \\
Cefadroxil & 70,000 & 3.7 \\
Cephalexin & 70,000 & 3.6 \\
\hline
\end{tabular}

specific reason-for-visit code associated with it), postoperative visit, and other growths of the skin (table 1).

In the NAMCS, HS was most often treated with a medication $(74 \%$ of visits) and much less often with a procedure (11\%; table 2$)$. The greatest number of patients received antibiotics, well ahead of pain medications and corticosteroids (table 3; fig. 2, 3). The systemic retinoid isotretinoin was also in the top ten.

Hidradenitis Suppurativa in the US

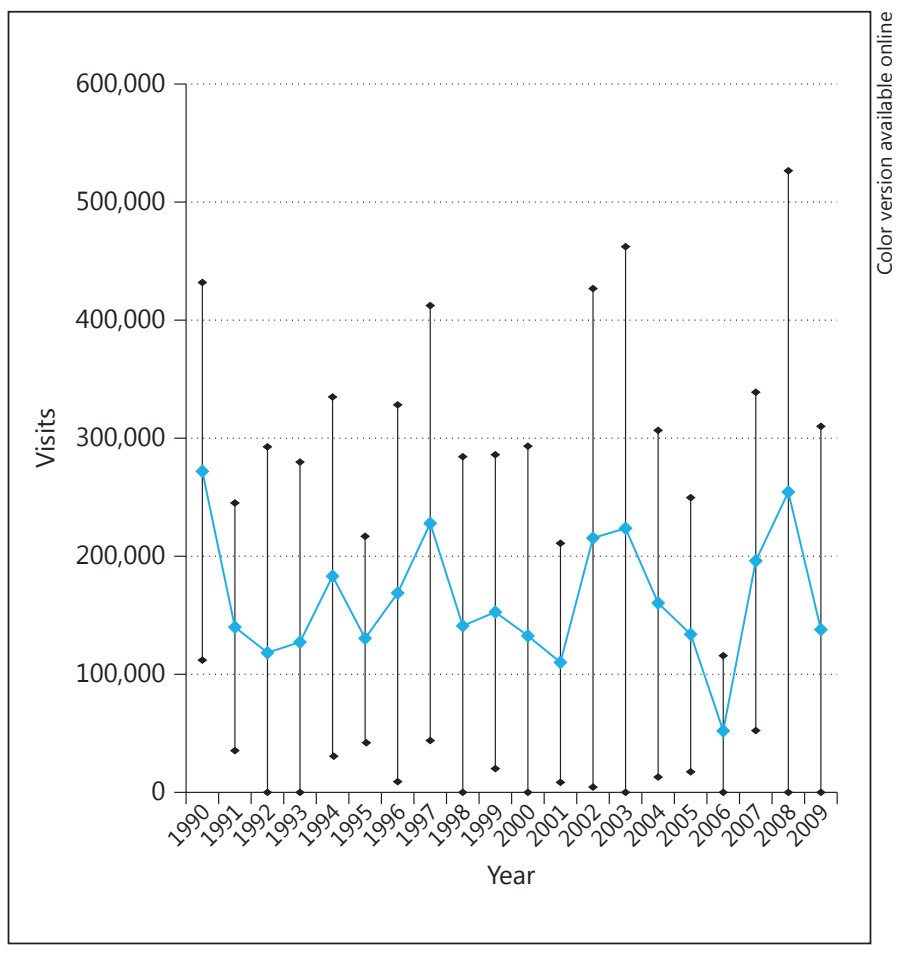

Fig. 1. Estimated NAMCS visits for HS in each year with vertical bars showing the 95\% CI. Most years had between 100,000 and 200,000 visits.

Systemic steroids prescribed included prednisone ( $0.4 \%$ of visits), methylprednisolone $(0.6 \%)$, and dexamethasone $(0.1 \%)$. No patients were observed to have been treated with tumor necrosis factor inhibitors (etanercept, adalimumab, or infliximab), or with the systemic treatments methotrexate, acitretin, or cyclosporine.

Although the sample size was not large enough to achieve statistical significance, patients appeared to re- 


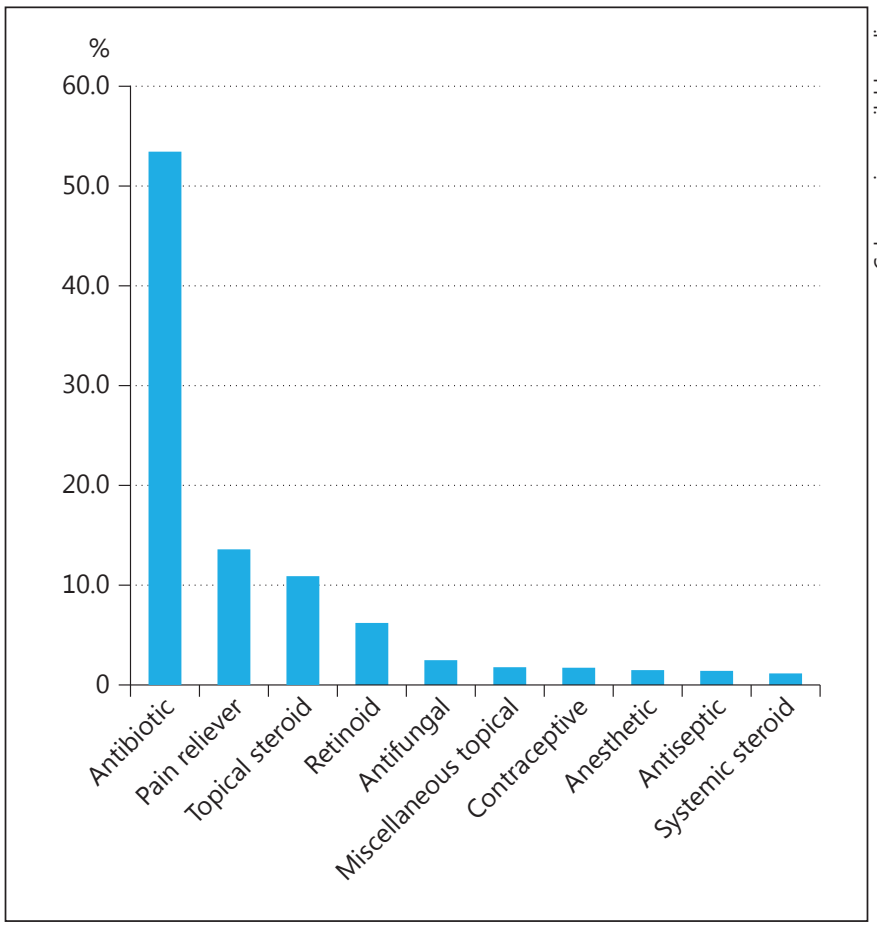

Fig. 2. Leading medication classes prescribed for the diagnosis of HS. Antibiotics were prescribed in more than half of HS visits in the NAMCS. Ten categories were prescribed in at least $1 \%$ of visits, and pain relievers and topical steroids were the only other categories prescribed in more than $10 \%$ of visits.

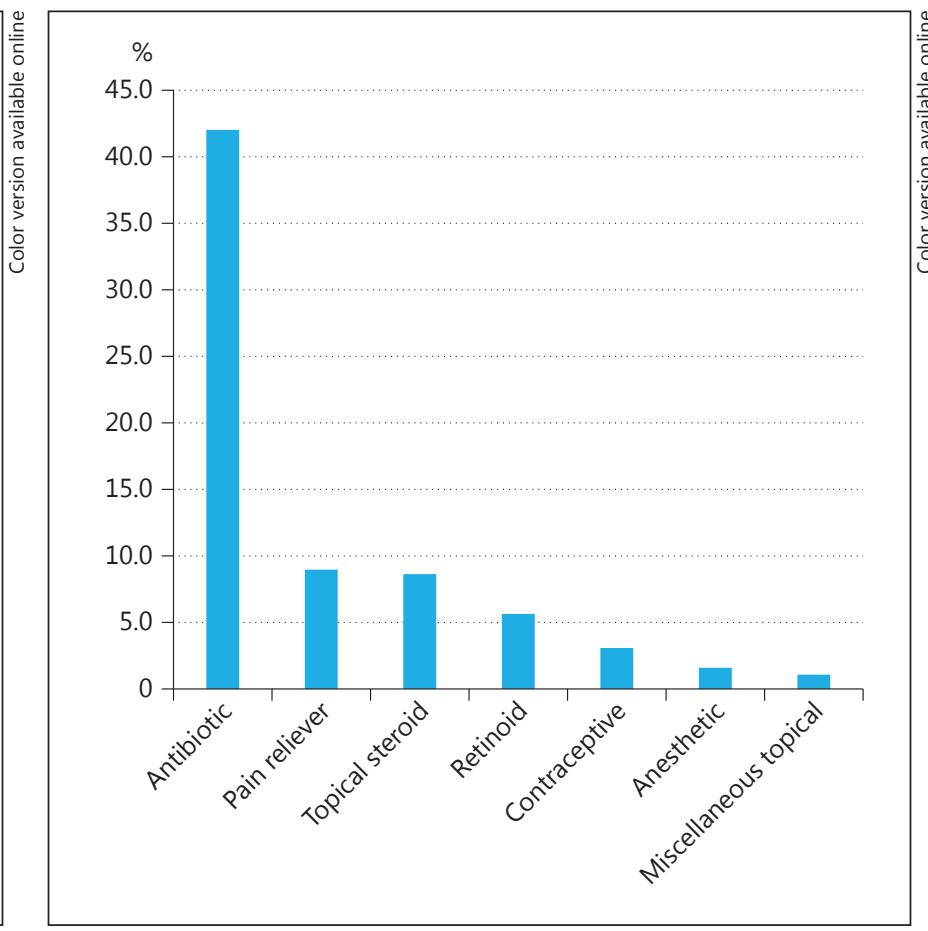

Fig. 3. Leading medication classes prescribed for the sole diagnosis of HS. Antibiotics were by far the most common treatments prescribed for visits with a sole diagnosis of HS in the NAMCS. Seven categories were prescribed in at least $1 \%$ of visits.

Table 4. Procedures done in visits for HS in the NAMCS

\begin{tabular}{lrc}
\hline Procedure (ICD-9 code) & Frequency & Visits, \% \\
\hline Local excision/destruction of lesion of skin (86.3) & 120,000 & 3.5 \\
Other incision with drainage of the skin (86.04) & 100,000 & 3.0 \\
Skin biopsy (86.11) & 30,000 & 1.0 \\
Other mammography (87.37) & 30,000 & 1.0 \\
Injection or tattooing of skin lesion or defect (86.02) & 30,000 & 1.0 \\
Injection or infusion of other therapeutic or prophylactic substance (99.29) & 30,000 & 0.9 \\
Application of other wound dressing (93.57) & 30,000 & 0.9 \\
Incision and drainage of scrotum and tunica vaginalis (61.0) & 20,000 & 0.6 \\
Open biopsy of soft tissue (83.21) & 10,000 & 0.4 \\
Nonoperative removal of other therapeutic device (97.89) & 10,000 & 0.3 \\
\hline
\end{tabular}

ceive combination therapy with a medication and a procedure more often when visiting dermatologists (15 vs. $6 \%)$. On the other hand, nondermatologists appeared more likely to give neither medication nor procedural treatment ( 28 vs. $2 \%)$.
When a procedure was done in the NAMCS, it was most often local excision or destruction of a skin lesion, or another incision with drainage of skin (table 4).

In MarketScan, the leading medications also included pain relievers and antibiotics, led by hydrocodone/acet- 


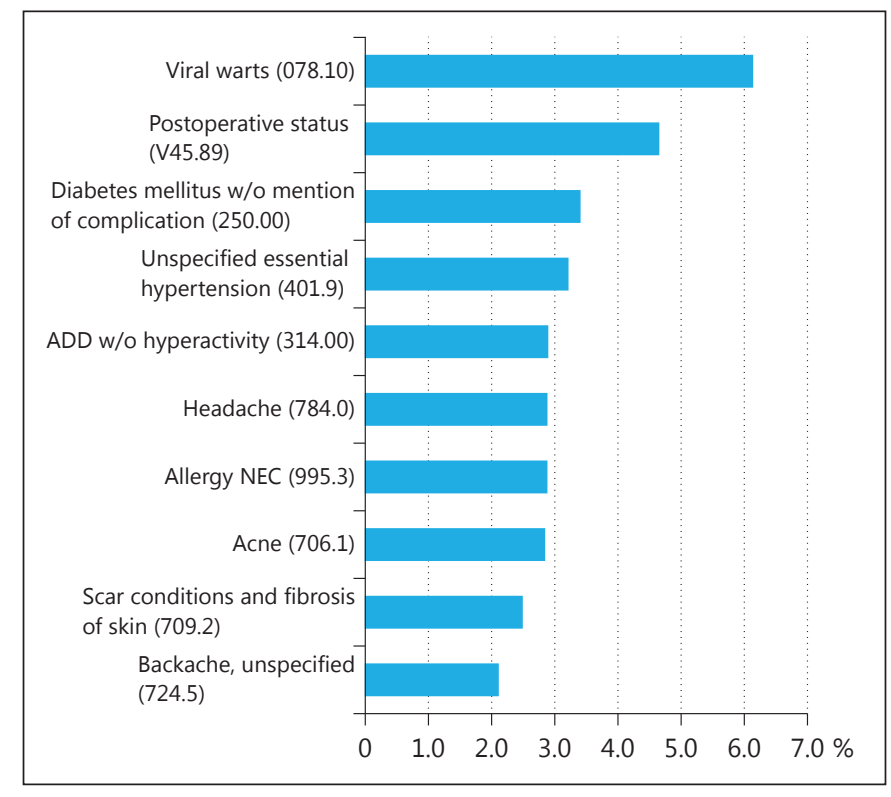

Fig. 4. Leading comorbidities in NAMCS patient visits for HS. The 10 comorbidities listed each occurred in at least $2 \%$ of visits. $\mathrm{ADD}=$ Attention deficit disorder; NEC $=$ not elsewhere classified.

Table 5. Leading medications prescribed in MarketScan in patients with HS

\begin{tabular}{lll}
\hline Name of medication & $\begin{array}{l}\text { Frequency } \\
\text { (patients) }\end{array}$ & Percent \\
\hline Hydrocodone/acetaminophen & 8,197 & 51.37 \\
Cephalexin & 7,765 & 48.67 \\
Ibuprofen & 5,385 & 33.75 \\
Sulfamethoxazole/trimethoprim & 4,965 & 31.12 \\
Doxycycline hyclate & 3,943 & 24.71 \\
Amoxicillin-potassium clavulanate & 3,887 & 24.36 \\
Oxycodone/acetaminophen & 3,664 & 22.96 \\
Amoxicillin & 3,589 & 22.49 \\
Albuterol & 2,999 & 18.80 \\
Ciprofloxacin HCL & 2,939 & 18.42 \\
\hline
\end{tabular}

All medications comprising more than $5 \%$ of the total are listed. These medications represent all claims made after the first diagnosis of HS. HCL = Hydrochloride.

aminophen and cephalexin (table 5). Etanercept, adalimumab, infliximab, methotrexate, cyclosporine, and acitretin did not appear among the top 200 treatments.

Antibiotics were by far the leading treatment listed in NAMCS visits for HS, whereas hydrocodone/acetamino- phen and ibuprofen were near the top of the list of MarketScan medications for HS. Since MarketScan records the prescriptions actually filled, while NAMCS records what was prescribed at the office visit, it may be that prescriptions for pain medications are more likely to be filled than those for antibiotics.

Complicating factors for comparison of the two datasets include sampling of office visits in NAMCS versus records of claims in MarketScan; tracking individual patients over time in MarketScan but not in NAMCS; analysis of only Medicaid patients in MarketScan versus patients with any payer in NAMCS, and the fact that MarketScan captures medications prescribed in emergency departments while NAMCS does not.

Leading procedures related to HS in MarketScan included incision and drainage of abscess, excision of axillary skin for hidradenitis with simple/intermediate repair, incision and drainage of abscess (multiple), and excision of axillary skin for hidradenitis with complex repair (table 6).

In NAMCS patients with HS, the leading comorbidities (fig. 4) included a variety of dermatologic conditions, such as warts and acne, as well as nondermatologic conditions such as diabetes and hypertension.

Patient visits for $\mathrm{HS}$ in the NAMCS showed a female:male ratio of about $2: 1$. The estimated percentage of female patients was $67.4 \%$ (95\% CI: 56.3-78.5). There was no statistically significant trend over time in gender distribution ( $\mathrm{p}>0.05)$.

In MarketScan, female patients with HS outnumbered male patients by nearly $5: 1$, making up $82.4 \%$ of the patients. MarketScan Medicaid patients overall are 58.4\% female; thus, HS patients are more than three times as likely to be female as MarketScan patients in general. Considering the NAMCS data, patients with HS on Medicaid were only $49 \%$ female, while those with a private insurance were $63 \%$ female and those on Medicare were $88 \%$ female.

Taking into account race and ethnicity, NAMCS patients with HS were $78.0 \%$ white, $17.5 \%$ black or African American, and $4.5 \%$ of unknown race (fig. 5). An estimated $4.9 \%$ were Hispanic or Latino of any race. Based on the 2000 Census estimates for the population, African Americans made slightly more than $50 \%$ more visits per 100,000 population than non-Hispanic Whites, while Hispanics made about half as many as non-Hispanic Whites (table 7). The difference in prevalence between Whites and African Americans may be greater than this, since African Americans make significantly fewer visits to dermatologists than Whites [5]. 
Table 6. Leading procedures performed in patients with HS in MarketScan

Description

Office or other outpatient visit for the evaluation and management of an established patient

Office or other outpatient visit for the evaluation and management of an established patient with a problem-focused history and examination, and straightforward medical decision making

Emergency department visit for the evaluation and management of a patient, which requires these 3 key components: an expanded problem-focused history; an expanded problem-focused examination, and medical decision making of moderate complexity Office or other outpatient visit for the evaluation and management of an established patient, which requires at least 2 of these 3 key components: a detailed history, a detailed examination, and medical decision making of moderate complexity

Incision and drainage of abscess - simple or single

Evaluation and management code for an emergency department visit, level 2

Culture, bacterial; any other source except urine, blood or stool, with isolation and presumptive identification of isolates

Anesthesia for procedures on the integumentary system on the extremities, anterior trunk and perineum; not otherwise specified

Complete blood count, with differential white blood cell count, automated

Excision of skin and subcutaneous tissue for hidradenitis, axillary; with simple or intermediate repair

New patient; detailed history, exam, straightforward decision making; $30 \mathrm{~min}$

Surgical pathology, gross and microscopic examination (per biopsy w/maximum of 4

per colposcopy, or maximum of 3 w/ECC [57505] or EMB [58110])

Collection of venous blood by venipuncture

Office consultation for a new or established patient (level 3)

Office consultation for a new or established patient (level 2)

Incision and drainage of abscess - complicated or multiple

New patient; expanded history, exam, straightforward decision making; $20 \mathrm{~min}$

Smear, primary source with interpretation; Gram or Giemsa stain for bacteria, fungi or cell types

Susceptibility studies, antimicrobial agent, agar dilution method, per agent

Level III surgical pathology, gross and microscopic examination skin cyst/tag/

debridement and others

All-inclusive clinic visi

Basic metabolic panel (laboratory)

Emergency visit outpatient: straightforward medical decision making

Emergency department visit for the evaluation and management of a patient with a detailed history and examination

Urine pregnancy test, by visual color comparison methods

Aerobic isolate, additional methods required for definitive identification, each isolate

Comprehensive metabolic panel (laboratory)

Excision of skin and subcutaneous tissue for hidradenitis, axillary; with complex repair

Injection, fentanyl citrate, $0.1 \mathrm{mg}$

Office or other outpatient visits for the evaluation and management of an established

patient that may not require the presence of a physician
Procedure

(CPT code)

Frequency

(patients)

99213

5,285

99212

2,330

13.49

99283

2,272

13.16

99214

1,695

9.81

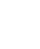

$\begin{array}{lll}10060 & 1,299 & 7.52 \\ 99282 & 1,151 & 6.66 \\ 87070 & 1,082 & 6.27\end{array}$

\begin{tabular}{lcc}
00400 & 1,044 & 6.05 \\
85025 & 962 & 5.57 \\
11450 & 890 & 5.15 \\
\hline
\end{tabular}

\begin{tabular}{lll}
99203 & 861 & 4.99 \\
88305 & 856 & 4.96 \\
\hline 36415 & 834 & 4.83 \\
\hline 99243 & 805 & 4.66 \\
\hline 99242 & 788 & 4.56 \\
\hline 10061 & 713 & 4.13 \\
\hline 99202 & 676 & 3.91 \\
\hline 87205 & 631 & 3.65
\end{tabular}

\begin{tabular}{lll}
87186 & 620 & 3.59 \\
\hline 88304 & 577 & 3.34 \\
& & \\
\hline $\mathrm{T} 1015$ & 520 & 3.01 \\
\hline 80048 & 482 & 2.79 \\
\hline 99281 & 476 & 2.76 \\
\hline 99284 & 468 & 2.71 \\
& & \\
\hline 81025 & 429 & 2.48 \\
\hline 87077 & 415 & 2.40 \\
\hline 80053 & 394 & 2.28 \\
\hline 11451 & 383 & 2.22 \\
\hline $\mathrm{J} 3010$ & 370 & 2.14 \\
\hline 99211 & 368 & 2.13
\end{tabular}

These include all procedures performed after the first HS diagnosis. The codes in italics are those that would be indicated for HS. $\mathrm{ECC}=$ Endocervical curettage; $\mathrm{EMB}=$ endometrial biopsy. 


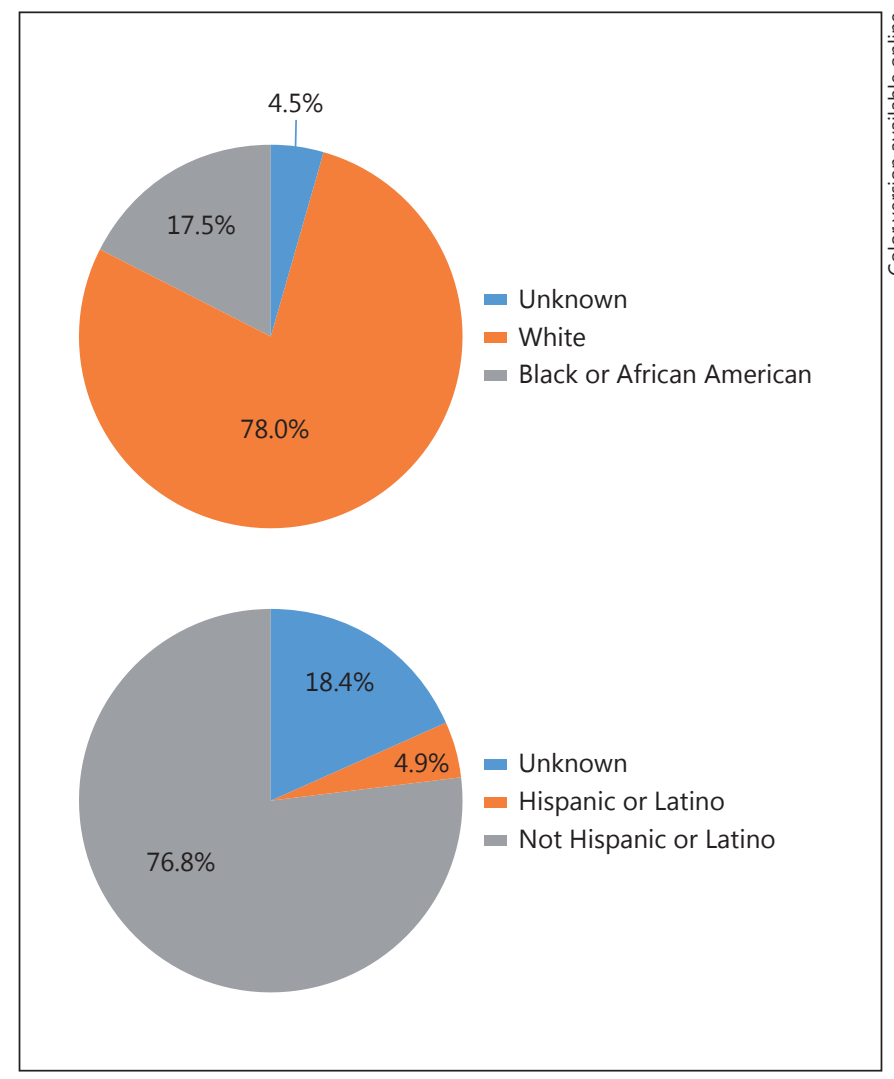

Fig. 5. A substantial percentage of HS visits in the NAMCS were by African American patients, while Hispanic or Latino patients made up a smaller percentage, although some of the visits with unrecorded ethnicity may have been Hispanic or Latino. a NAMCS patient visits for HS by race. b NAMCS patient visits for HS by ethnicity.

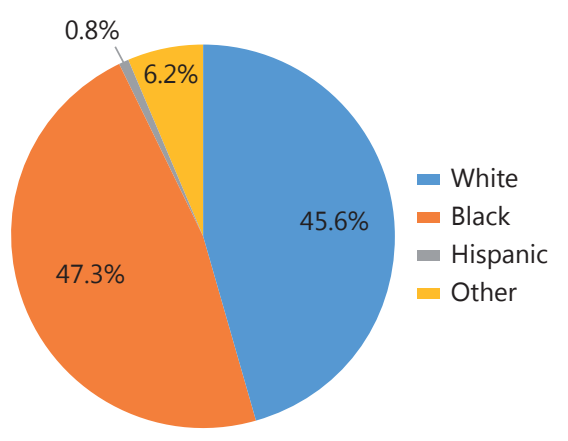

Fig. 6. Race/ethnicity of MarketScan patients with HS. MarketScan patients with HS were most frequently black, and $6 \%$ were neither white, black, nor Hispanic.

Hidradenitis Suppurativa in the US

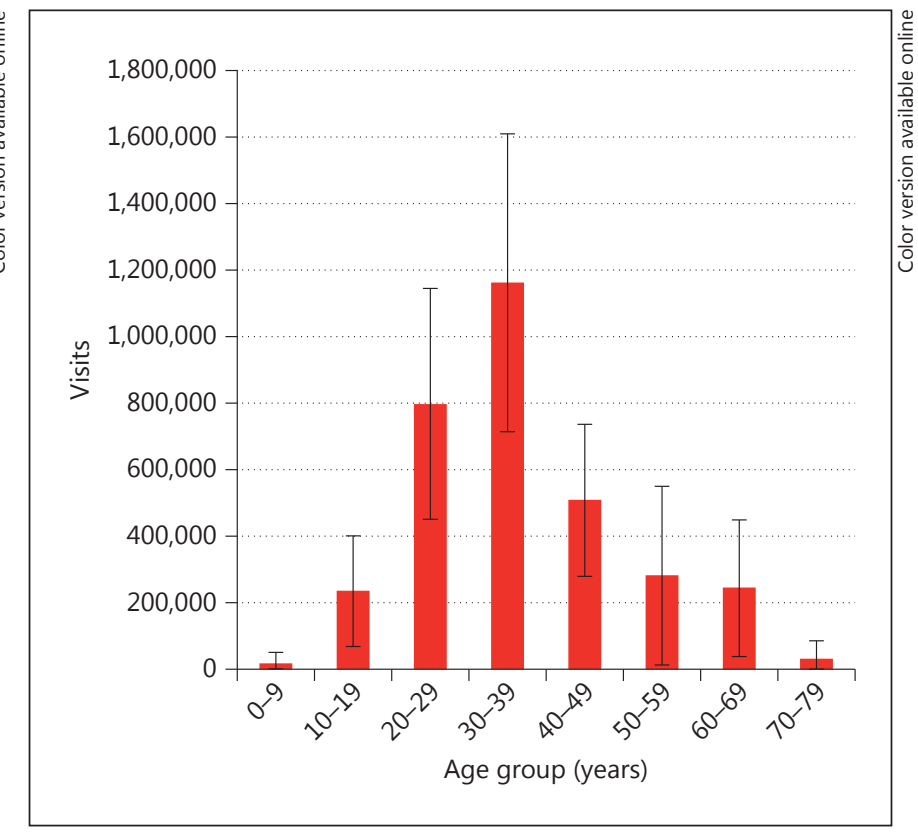

Fig. 7. Number of NAMCS visits for HS by age group with $95 \%$ CI. Thick bars show the estimated number of visits; thin vertical bars present the $95 \%$ CI for visits in each age group.

MarketScan patients with HS (fig. 6) were $45.6 \%$ nonHispanic white, $47.3 \%$ black, $0.8 \%$ Hispanic, and $6.2 \%$ other races. This distribution reflects the general racial and ethnic distribution of the Medicaid population studied in MarketScan data, which is $52.2 \%$ non-Hispanic White, $31.2 \%$ Black, $4.6 \%$ Hispanic, and $12.0 \%$ other races. The ratio of blacks to whites is 1.74 times as high for HS patients compared to MarketScan patients in general.

In the NAMCS, HS patients were mostly young adults, with the greatest number of visits by patients in their 30s, followed by those in their 20s (fig. 7). These age groups accounted for about $60 \%$ of all HS visits. There was no statistically significant trend over time in average age $(\mathrm{p}>0.05)$. In MarketScan, the greatest number of patients were in their 20 s, followed closely by those in their $30 \mathrm{~s}$ (fig. 8), with a steady decline across the older groups. In MarketScan, we used the age at first HS diagnosis, which may have contributed to MarketScan patients being somewhat younger.

Physicians of several specialties commonly saw HS patients in the NAMCS (fig. 9). Almost equal numbers of patients visited general and family physicians (24.2\%), general surgeons $(22.6 \%)$, and dermatologists $(22.6 \%)$. On a per physician basis, dermatologists managed the most cases per year, with about 4 cases per physician per 


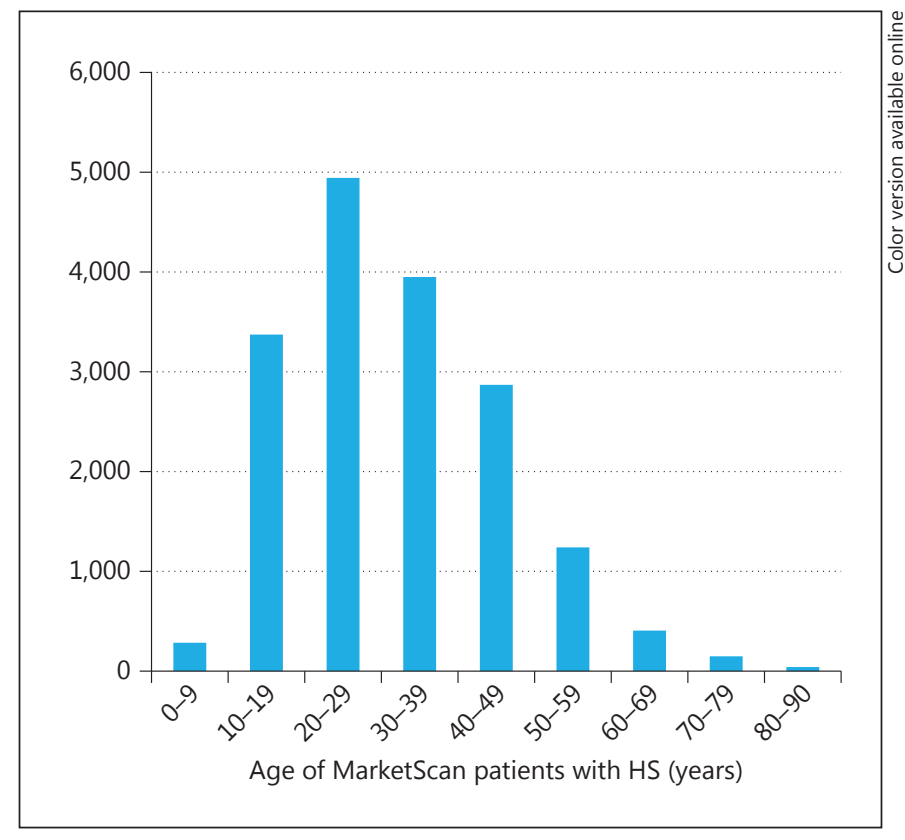

Fig. 8. MarketScan patients with HS by age group. In this population, HS diagnoses peaked in the 20 s with a steady decline thereafter.

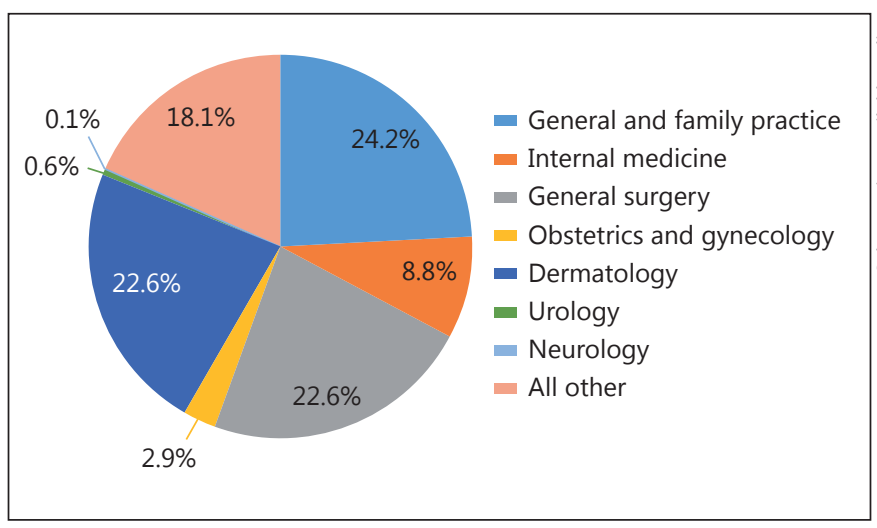

Fig. 9. NAMCS patient visits for HS by physician specialty. The patients were about equally likely to see a general or family physician, general surgeon, or dermatologist.

Table 7. NAMCS annual visits per 100,000 Americans of each race and ethnicity based on the 2000 Census data

\begin{tabular}{ll}
\hline White, not Hispanic or Latino & 48 \\
Black or African American & 78 \\
Hispanic or Latino & 22 \\
\hline
\end{tabular}

HS appears to affect African American patients most often. year. General surgeons managed about 2 cases per physician per year, while the average general and family physician managed 0.5 cases. It appears that while dermatologists have somewhat more experience, no specialty has a great deal of experience treating this condition.

\section{Discussion}

The number of HS visits was quite low, considering a reported prevalence of $1 \%$, or as much as $4 \%$ for the peak age group, in the literature [1]. Recent estimates based on nationally representative data by McMillan [6] and Cosmatos et al. [7] have challenged the previous estimates and suggest that the prevalence may be significantly lower. McMillan speculates that the high prevalence previously reported is likely due to a self-report questionnaire with relatively low specificity, causing as much as $94 \%$ of identified cases to be false positives [6]. A local populationbased study of the incidence in Olmsted County, Minn., USA, also supported the idea that HS is rather rare [8].

With an annual estimate from the NAMCS of 164,000 visits per year, a maximum of around $0.06 \%$ of the population is being treated at any given time. It may be that the prevalence is higher than the NAMCS would suggest, but many sufferers are not getting any treatment. Alternatively, cases of HS may be getting coded as something other than 705.83, or they may be misdiagnosed.

The data do support the idea that the typical HS patient is a female in her 20s or 30s, who is likely to be African American. This group may suffer from a particularly large quality of life impact from HS symptoms and should welcome new treatment options. The demographics also underscore the importance of availability of HS treatments that are safe for women who may become pregnant. Treatments such as acitretin and methotrexate are teratogenic or fetotoxic, but biologics are relatively safe during pregnancy (category B) and may have a good chance to become commonly used [9].

Limitations of the NAMCS include the fact that it includes only outpatient visits to nonfederally employed providers and does not permit following individual patients over time. The standard unit of analysis is the patient visit. The severity of the disease and the body site are not recorded in the NAMCS, except insofar as the body site may occasionally be inferred from the patient's reason for visit. In patients with multiple diagnoses, it is not always possible to determine which medication was prescribed to treat which condition. Nevertheless, the extensive information gathered on each visit allows for the 
assessment of demographics, differences between the experiences of particular groups, and trends over time.

The data seem to suggest that systemic treatments, other than antibiotics and pain medications, are rarely used at present. Some isotretinoin is used, but isotretinoin is no longer believed to be effective for HS. The available MarketScan data only go up to 2007, and the published trials of biologics for HS are all from 2010 or later, so it may be that the current datasets do not capture the most recent changes in practice. However, the fact that the average MarketScan patient with HS got approximately 12 different medications seems to suggest that existing treatments have not been very effective. Therefore, although the HS population appears relatively small, there may be a high demand for data on the safety and efficacy of biologics for treating the disease in this population.

\section{Acknowledgments}

The Center for Dermatology Research is supported by an unrestricted educational grant from Galderma Laboratories, L.P. Dr. Feldman has received grants from Galderma, Astellas, Abbott
Labs, Warner Chilcott, Janssen, Amgen, Photomedex, Genentech, BiogenIdec, Coria/Valeant, Pharmaderm, Ortho Pharmaceuticals, Aventis Pharmaceuticals, Roche Dermatology, 3M, Bristol Myers Squibb, Stiefel/GlaxoSmithKline, Novartis, Medicis, Leo, HanAll Pharmaceuticals, Celgene, Basilea, and Anacor.

\section{Statement of Ethics}

This study was declared exempt by the Wake Forest Baptist Hospital Institutional Review Board and the University of Michigan Institutional Review Board.

\section{Disclosure Statement}

Dr. Feldman is a consultant and speaker for Galderma, Stiefel/ GlaxoSmithKline, Abbott Labs, Warner Chilcott, Janssen, Amgen, Photomedex, Genentech, BiogenIdec, and Bristol Myers Squibb and has received stock options from Photomedex. Moreover, Dr. Feldman is the founder and holds stock in Causa Research. Mr. Davis, Dr. Lin, and Dr. Balkrishnan have no conflicts to disclose.

\section{References}

1 Jemec GB: Clinical practice. Hidradenitis suppurativa. N Engl J Med 2012;366:158-164.

2 Grant A, Gonzalez T, Montgomery MO, Cardenas V, Kerdel FA: Infliximab therapy for patients with moderate to severe hidradenitis suppurativa: a randomized, doubleblind, placebo-controlled crossover trial. J Am Acad Dermatol 2010;62:205-217.

3 Miller I, Lynggaard CD, Lophaven S, Zachariae C, Dufour DN, Jemec GB: A double-blind placebo-controlled randomized trial of adalimumab in the treatment of hidradenitis suppurativa. Br J Dermatol 2011;165:391-398.

4 Adams DR, Yankura JA, Fogelberg AC, Anderson BE: Treatment of hidradenitis suppu- rativa with etanercept injection. Arch Dermatol 2010;146:501-504.

5 Davis SA, Narahari S, Feldman SR, Huang W, Pichardo-Geisinger RO, McMichael AJ: Top dermatologic conditions in patients of color: an analysis of nationally representative data. J Drugs Dermatol 2012;11:466473.

6 McMillan K: Hidradenitis suppurativa: number of diagnosed patients, demographic characteristics, and treatment patterns in the United States. Am J Epidemiol 2014;179: 1477-1483.

7 Cosmatos I, Matcho A, Weinstein R, Montgomery MO, Stang P: Analysis of patient claims data to determine the prevalence of hidradenitis suppurativa in the United States. J Am Acad Dermatol 2013;68:412-419.

8 Vazquez BG, Alikhan A, Weaver AL, Wetter DA, Davis MD: Incidence of hidradenitis suppurativa and associated factors: a populationbased study of Olmsted County, Minnesota. J Invest Dermatol 2013;133:97-103.

9 Menter A, Gottlieb A, Feldman SR, Van Voorhees AS, Leonardi CL, Gordon KB, et al: Guidelines of care for the management of psoriasis and psoriatic arthritis: section 1 . Overview of psoriasis and guidelines of care for the treatment of psoriasis with biologics. J Am Acad Dermatol 2008;58:826-850. 